\title{
The role of Smith chart in teaching the course of "Microwave Technology and Antenna"
}

\author{
Xuejun Zhang \\ School of Computer and Electronic Information \\ Guangxi University \\ Nanning, Guangxi 530004, P. R. China \\ e-mail:xjzhang@gxu.edu.cn
}

\author{
Jinwen Deng \\ School of Defense Force and Education \\ Guangxi University \\ Nanning, Guangxi 530004, P. R. China \\ e-mail: 420710294@qq.com
}

\begin{abstract}
Due to the difficulties in learning and teaching microwave technology and antenna, we introduced the Smith chart as a computer-aided experimental teaching method to improve the teaching quality. By using the computer language for calculation and visualization of the parameters of microwave on transmission line, the teaching reform effect is evaluated in compare with the students' scores within 3 years. The results show that the teaching reform is promising to help students in the understanding of the basic concepts of microwave.
\end{abstract}

Keywords- Microwave technology; Computer aided teaching, Smith chart; Teaching reform

\section{INTRODUCTION}

With the rapid development of modern communication technology, the carrier frequency using in modern systems developed more quickly than the traditional technologies that are always referred to MHZ band. Today, we have entered the thousand MHZ band eras, so-called the microwave band, on which plays an important role in the design and manufacture of a cell phone, the development of antenna for mobile communication and so on. Both Microwave technology and antenna technology have become more and more important in every industry, even in people's daily life. Therefore, it is essential to improve college students' ability of designing the microwave wireless communication system that should lead to enhance their employability. The course of "microwave technology and antenna" is an important professional foundation course to the students of both electronics and communication engineering which helps students to master the basic concepts, theory and analysis method of microwave technology; trains the students' ability to analyze and solve the communication problems which can lay a foundation for their future job engaging in the research of microwave - the design and manufacturing of communication system, circuits, and equipments. In addition, it is also indispensable to the subsequent courses such as "mobile communication". As for electronics and communication engineering students, this type of courses such as "electromagnetic field and wave", "microwave technology and antenna" are indispensable for current and future [1-3]

Generally, it is a tough work for students to understand and master this course for its strong theoretical property, complicated and abstract content, various analysis methods, and higher demands for mathematical knowledge. Based on the difficulties in teaching and learning of microwave technology and antenna course, this study aims at improving the teaching quality from the most basic parameter concept and calculation of microwave by introducing a computer programming experiment - Smith chart that helps the students to understand the various concepts in microwave transmission line, such as the input impedance, admittance, reflection coefficient, and so on.

Smith chart is an effective method used to analysis matching problems of transmission line. This experiment asks students to design the computer aided software of Smith chart by referring to the materials in their textbooks and covering the calculation of input impedance, admittance and impedance matching of the microwave transmission line. It can not only avoid tedious formula and complex operations which result in a simple calculation in the engineering design, but also make the physical conception clearer in graphic processing as the outcome is visualized. The effectiveness of this teaching reform is verified by comparing to the scores of the past two years without Smith chart experiment.

\section{FEATURES OF THE COURSE}

The course of "microwave technology and antenna" deals with microwave technology, antenna and wave propagation, microwave application system, and so on. It has the following characteristics:

\section{A. Strong theoretical}

This course is an extension of the theory of electromagnetic field and wave, in which three-dimensional vector field is studied for building up various field distribution through the analysis of vector divergence, curl and boundary conditions. Maxwell equations, as a clue throughout the course, are used to deduce the transmission characteristics of electromagnetic wave in ideal transmission line, guided wave system, and free space. Furthermore, the requirement of determination of the radiation fields with different shapes of antenna asks students to master the higher mathematics knowledge and matrix theory [4].

\section{B. Abstract and complex concepts}

Waveguide theory includes the physical structure and working principle of rectangular waveguide, circular waveguide, coaxial line, whose field distributions are in three dimensional. The same situation also occurs in the 
microwave components such as power distribution components, resonator and all kinds of connections and matching components. Students are required to have certain space imagination ability and abstract thinking ability to understand all kinds of plane antennas, wire antenna structure, feedback principle, radiation directional diagram, and so on.

\section{Stronger Application and practicality}

This course has many experiments, such as: Smith chart experiment, microwave measurement, etc. It has a widely application in many systems, such as radar, navigation, remote sensing, microwave communication and deep space communication system.

\section{LIMITATIONS IN THE PAST TEACHING ACTIVITIES}

\section{A. The teaching mode and method}

Traditional unitary teaching mode usually goes on only with a book, a blackboard and a piece of chalk, those might be hard to improve the efficiency of the lecture as the teachers waste so much time in writing on the board; too much emphasis on theory learning; less practice teaching throughout the limited teaching time in class [5].

\section{B. Former students' scores}

As illustrated in table 1, the previous teaching achievements and academic records are not optimistic with the failing rate up to $10 \%$. Investigation is undergone by accounting for the scores of examination and students' response. The results indicated that the students' foundation on this course was unreliable and some concepts were not profoundly understood, especially the problems related with impedance matching when Smith chart was used to solve transmission line parameters and impedance matching.

\section{A PRELIMINARY PRACTICE OF TEACHING REFORM}

\section{A. The Classroom Section}

Considering the characteristics of strong theoretical, abstract and complicated in "microwave technology and antenna" course, the teacher combines the traditional classroom mode with the modern multimedia teaching methods to provide convenience of question and answer activities at class. Using the form of discussion and interactive processes makes the curriculum statement vivid with a large amount of information and saves time as well. Teachers can explain the profound course and create a good learning atmosphere at the same time. According to the teaching plan, the exercise lessons and class discussions are also brought into the classroom which forces students to puzzle out the questions they meet in their homework. Making full use of network resources to enrich teaching content, teacher also answers a host of questions remotely, via Internet, or on the phone in consideration of the convenience and time-saving. Meanwhile, introducing several teaching blogs with the electronic materials and media presentation materials stimulates students' enthusiasm for learning. Furthermore, encouraging students to find the latest related research progress and the current research hot spot problems ensures them an independent exploration, independent learning, mutual discussion and self innovation learning.

\section{B. Experimental Section}

In this course, the experiment of Smith chart is a basic experiment which plays a pivotal role that helps students to understand Smith chart itself and other basic concepts on microwave, such as transmission line, impedance matching, characteristic impedance of the coaxial line, etc. throughout the course. The experiment is arranged after learning $\lambda / 4$ wavelength impedance matching method and short-circuited stub line method. According to the state analysis of the transmission line and formula calculation, the experiment requires the realization of parallel branch stub matching method which is generally abstract and complicated for students. Thus the Smith chart experiment can provide a reference point for test by selecting the impedance matching method.

Under the teacher's careful preparation and arrangement, students have the readiness lesson before experiment. In computer room, for the sake of initiating student into the craft, the basic operation of the software, basic programming ideas and basic methods using in Microsoft Visual C\# 2005 is taught by the computer programming teacher we invited. From industry experts to beginners or students, this software is easy to use and study because of its powerful functions, simple manipulation and visualization. Under the programming the teacher's guidance, the students have a preliminary understanding and mastering about Visual $\mathrm{CH}$, resulting in their ability to complete a project in creating Windows form a circle, arc, linear operation independently. After two experimental preparation lessons, students already grasp the basic programming method of Microsoft Visual C\# 2005 before they are required to finish the Smith chart experiment within three weeks. The experiment involved in some complicated formula algorithms that ask the students to be familiar with the principle of the Smith chart, which is comparatively complex, abstract for beginners. They need to spend some time in learning and understanding since only after mastering each formula of parameters can the students develop the Smith chart into a program which can run on computer. For example, we ask students to obtain the impedance value in the Smith chart by simply moving the mouse to change a point coordinates $(a, b)$, and they should find out the relation from the equation between coordinate and resistance/reactance circle:

$$
z=r+j x=-\frac{a^{2}+b^{2}-1}{(a-1)^{2}+b^{2}}+j \frac{2 b}{(a-1)^{2}+b^{2}}
$$

Only if well understanding of this equation can they calculate $r, x$ from $a, b$, then further capability of drawing a 


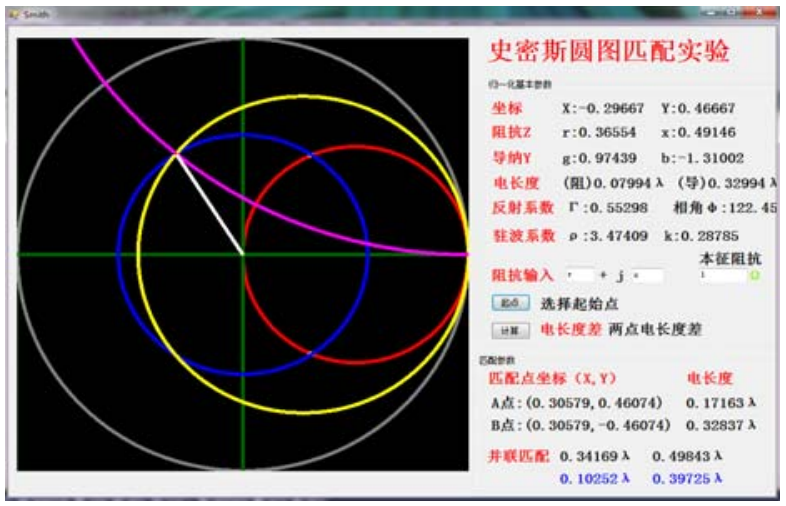

Figure1. The user interface of Smith chart for computer aided teaching.

resistance circle at center $\left(\frac{r}{r+1}, 0\right)$ with radius of $\frac{1}{r+1}$, and a reactance circle at center $\left(1, \frac{1}{x}\right)$ with radius of $\frac{1}{x}$.

After three weeks' exploration, self study and self innovation, students hand in their homework. In the class, the teacher gives a reference answer as shown in figure 1 . The programming steps are explained in details with demonstrations and presentations on each examples appeared in the textbook to verify the problems about input impedance, admittance and impedance matching. During these procedures, the students have a clear understanding of the reference samples they have already calculated before. We summary the significance of Smith chart experiment as the following points:

- It promotes students to master an overall understanding of the Smith chart and other important knowledge of microwave.

- As we all know, Smith chart is such an important part of microwave technology in engineering application

Table 1 Comparison Results of W/O Smith Chart Experiment within Three Academic Years)

\begin{tabular}{|c|c|c|c|c|c|c|}
\hline \multicolumn{7}{|c|}{ 2007-2008 Session (without Smith Chart) } \\
\hline \multicolumn{3}{|c|}{ Exam Form: Close Exam } & \multicolumn{2}{|c|}{ Exam Date: 2008.7} & \multicolumn{2}{|l|}{ Students: 63} \\
\hline \multirow{4}{*}{ 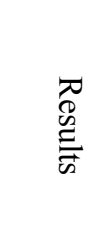 } & Grads & 90-100 Excellent & $80-89$ Good & 70-79 Medium & $60-69$ Pass & $<60$ Failed \\
\hline & Students & 11 & 15 & 16 & 10 & 11 \\
\hline & Percentage & $17.46 \%$ & $23.81 \%$ & $25.40 \%$ & $15.87 \%$ & $17.46 \%$ \\
\hline & Average & & 72.23 & Standard Deviation & & 24.77 \\
\hline
\end{tabular}

\begin{tabular}{|c|c|c|c|c|c|c|}
\hline \multicolumn{7}{|c|}{ 2008-2009 Session (without Smith Chart) } \\
\hline \multicolumn{3}{|c|}{ Exam Form: Close Exam } & \multicolumn{2}{|c|}{ Exam Date: 2009.7} & \multicolumn{2}{|l|}{ Students: 55} \\
\hline \multirow{4}{*}{$\begin{array}{l}\text { Dृ } \\
\stackrel{\mathbb{E}}{\mathscr{E}} \\
\stackrel{\equiv}{\Rightarrow}\end{array}$} & Grads & 90-100 Excellent & $80-89$ Good & 70-79 Medium & $60-69$ Pass & $<60$ Failed \\
\hline & Students & 11 & 18 & 11 & 11 & 4 \\
\hline & Percentage & $20.00 \%$ & $32.73 \%$ & $20.00 \%$ & $20.00 \%$ & $7.27 \%$ \\
\hline & Average & & 75.75 & Standard Deviation & & 19.72 \\
\hline
\end{tabular}

\begin{tabular}{|c|c|c|c|c|c|c|}
\hline \multicolumn{7}{|c|}{ 2010-2011 Session (with Smith Chart) } \\
\hline \multicolumn{3}{|c|}{ Exam Form: Close Exam } & \multicolumn{2}{|c|}{ Exam Date: 2011.7} & \multicolumn{2}{|l|}{ Students: 52} \\
\hline \multirow{4}{*}{ 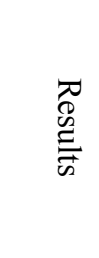 } & Grads & 90-100 Excellent & $80-89$ Good & $70-79$ Medium & $60-69$ Pass & $<60$ Failed \\
\hline & Students & 17 & 20 & 12 & 3 & 0 \\
\hline & Percentage & $32.69 \%$ & $38.46 \%$ & $23.08 \%$ & $5.77 \%$ & $0.00 \%$ \\
\hline & Average & & 84.27 & Standard Deviation & & 8.98 \\
\hline
\end{tabular}


that the students will make a huge difference in their future work if a better mastery and understanding of this analysis methodology are grasped.

- It is a bold attempt from the book knowledge to practical education, which does great help to facilitate students to have a renewed recognition of Smith chart and form a good practice in abstract concepts learning.

\section{Summary and evaluation}

After a semester period of teaching preliminary probe in the implementation of the education plan, the teaching quality has been improved significantly. Covering from the teachers' teaching experience or assignments' revising to the students' own understanding of the course, the results turn out to be satisfied. Especially, the final test scores of the course reflect the remarkable results of the curriculum reform after which students' final test scores have an obvious enhancement.

As illustrated in Table 1, the result shows an increase in an outstanding proportion from previous $20 \%$ to $32.69 \%$, and a decrease in the failed proportion form previous $10 \%$ to zero, while the average scores rose from 73 points to 84 points. In a word, such a result demonstrates that the educational reform at preliminary exploring is comparatively successful.

This experiment acquainted the students with Microsoft Visual C\# software programming meanwhile deepened their understanding of the Smith chart. At the same time, it instructed them to grope the intersection application of an unfamiliar interdisciplinary subject as well. Because students seldom involved with software programming toward such a practical problem in the past, they felt overwhelmed in the beginning when worked on those codes. After studying through internet search, referring to books, and so on, they gradually formed their own basic frame by exploring, and learned how to write codes for specific formula. Although not everyone reached an ideal Smith chart result, each of them felt happy and productive as they have learned a lot in this challenging practice.

\section{A trial of English teaching exploring}

With the rapid development of internationalization, it is essential for engineering students to catch up with the changing speed of the high gear on microwave technologies. We started a trial of English teaching exploring at 2011-2012 session. Considering the difficulty of this course, we selected a relative easier textbook, "electromagnetic field theory fundamentals" by Bhag Singh Guru [6], for the first reform trial. Because there is no content on the microwave network analysis on this book, a supplemental teaching material of "microwave engineering" by David M. Pozar [7] was also introduced to this course. Smith Chart experiment was retained in our teaching activity while some contents on the microwave circuits and systems were ignored because of the limitation of time and handicap of English language. The course of professional English with 40 academic hours was just finished at first part of 2012 session, in which students had grasped many basic vocabularies on electromagnetic fields and waves, before we started the microwave technology and antenna course at second part of 2012 session. The final testing results indicates that there is a little reversal with the rate of failed proportion to $6 \%$, and outstanding proportion to $17 \%$, but it is reasonable by taking account of a bilingual education. Our future work will be focused on how to improve the teaching effect of this course in English.

\section{CONCLUSIONS}

The microwave technology and antenna course has the characteristics of a strong theory, wide application, large contents, high complexity and difficult in teaching and learning. Based on the previous teaching experience and the author's new experience, this research provides a practical, exploratory methods and ideas regarding on how to improve the quality the microwave technology and antenna course teaching and the cultivation of the students' practice and innovative ability. It is expected that our exploring could give some helps to the teachers and the students who are working on this course.

\section{ACKNOWLEDGEMENT}

This research was supported in part by the National Natural Science Foundation of China (No. 61262027); in park by a research foundation project of the Guangxi Ministry of Education (No. 200810MS048).

\section{REFERENCES}

[1] WangXinWen, Li ping, LiYanPing. Microwave technology and antenna (second edition) [M]. Beijing: Electronics Industry Publication, 2008.

[2] LiuXueGuan, CaoHongLong, GuoHuiPing. Microwave technology and antenna (M). Xian:Xian University of Electronic Science and Technology Press, 2006

[3] LiuXueGuan, CaoHongLong, GuoHuiPing. The microwave technology and antenna: learning guide (second edition) (M). Xian: Xian University of Electronic Science and Technology Press, 2006.

[4] ChenYuQun, YangLiBo, CDC). The microwave technology and antenna course teaching reform exploration $[\mathrm{J}]$. Science and Technology Information, 2009 (24) : 216

[5] ZhengXueMei, WuChunMing, LeiYuLing. Discuss the microwave technology course teaching reform [J]. Acta Innovation of Science and Technology, 2008 (24) : 164

[6] Bhag Singh Guru, Huseyin R. Hiziroglu. Electromagnetic field theory fundamentals (second edition) $[\mathrm{M}]$. Beijing: China Machine Press, 2004.

[7] David M. Pozar. Microwave engineering (second edition) [M]. New York: John Wiley \& Sons, INC. 1998. 